\title{
Electromagnetic monitoring of the Earth's interior in the frame of the MEM Project
}

\author{
Paolo Palangio $\left({ }^{1}\right)$, Cinzia Di Lorenzo $\left({ }^{1}\right)$, Manuele Di Persio $\left({ }^{1}\right)$, Fabrizio Masci $\left({ }^{1}\right)$, \\ Spomenko Mihajlovic $\left({ }^{2}\right)$, Lucia Santarelli $\left(^{1}\right)$ and Antonio Meloni $\left({ }^{1}\right)$ \\ (') Istituto Nazionale di Geofisica e Vulcanologia, L'Aquila, Italy \\ $\left.{ }^{2}{ }^{2}\right)$ Geomagnetic Institute of Grocka, Beograd, Serbia
}

\begin{abstract}
The MEM Project (Magnetic and Electric fields Monitoring) has been running in the INGV Observatory of L'Aquila since 2004. The principal purpose of the project is to create a network of observatories in Central Italy to monitor the electromagnetic signals in the frequency band $[0.001 \mathrm{~Hz}-100 \mathrm{kHz}]$. This band includes natural signals (magnetic pulsations of magnetospheric origin, Earth-ionosphere resonance mode signals, atmospheric noise, and so on) and artificial signals (power line emissions, VLF radio transmissions, and so on). The innovative characteristic of the project is the approach chosen to study the complex problem concerning the representation of the spatial and temporal distributions of the electromagnetic fields in the band of interest. Both the distributions can be represented by some parameters containing the locations and the characteristics of the sources of the electromagnetic signals. When all the stations will be in operation the wide-band interferometry will be applied. Combining the simultaneous observations of the electromagnetic field measured in the stations of the network, we will be able to obtain detailed information about the investigated electromagnetic sources. A new measurement system has been developed to fulfil these requirements focusing on the automation of the measurements. The system is designed for long term recording of the electromagnetic fields in a wide frequency band. In the frequency band [1 Hz-100 kHz] the three components of the magnetic field and the three components of atmospheric electric field are processed in real time using DSP (Digital Signal Processing) techniques. In the frequency band [0.001-25] Hz the two components of the telluric field and the three components of the magnetic field are recorded as sampled $(100 \mathrm{~Hz})$. One of the main scientific objectives of the MEM project is the longterm monitoring of the geodynamical processes, such as the earthquakes, by the calculation of the Poynting vector, and the analysis of the magnetic transfer functions and impedance tensor. In the coming years this kind of analysis can be useful to underline the possible correlation between the geodynamical processes and the local magnetic field anomalies.
\end{abstract}

Key words MEM Project-electromagnetic sources - Earth's interior - geodynamical processes

\section{Introduction}

The main target of the MEM project is to create a network of permanent observatories in

Mailing address: Dr. Paolo Palangio, INGV, Osservatorio Geofisico di L'Aquila, Castello Cinquecentesco, 67100, L'Aquila, Italy; e-mail:palangio@ingv.it
Central Italy to monitor the environmental electromagnetic signals. The leader partner of the project is the Abruzzo Region; the other partners are the INGV (Italian Istituto Nazionale di Geofisica e Vulcanologia) Observatory of L'Aquila, the Regional Environmental Agency of Molise (ARPA-Molise), the University of Ferrara, the University of Tirana, Albania and the Geomagnetic Institute of Grocka, Beograd, Serbia. The scientific objectives of the MEM Project are the characterization of the electromagnetic background noise in the Earth-ionosphere cavity in the band of frequency [0.001 $\mathrm{Hz}-100 \mathrm{kHz}]$, the realization of a model of the 


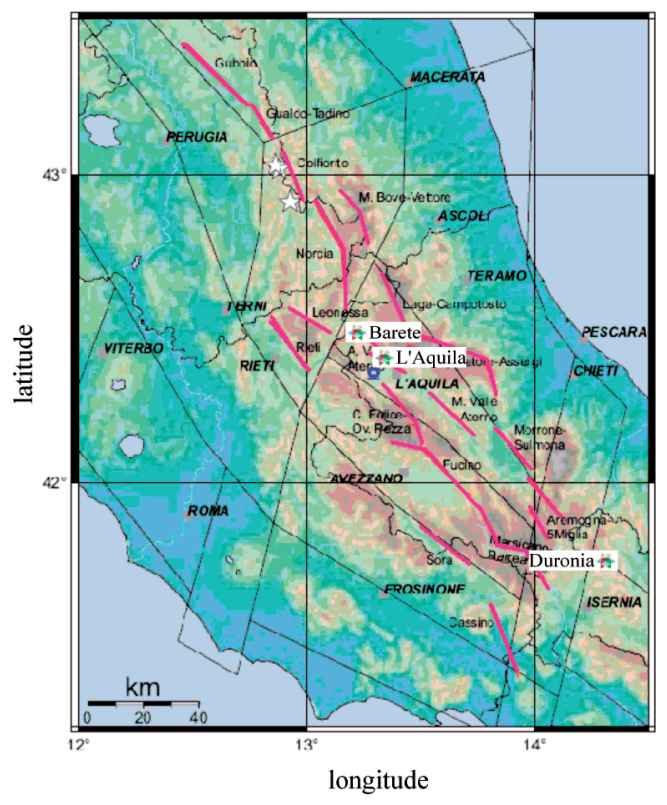

Fig. 1. The locations in Central Italy of the first three MEM stations. The faults distribution in the Central Apennines is also shown.

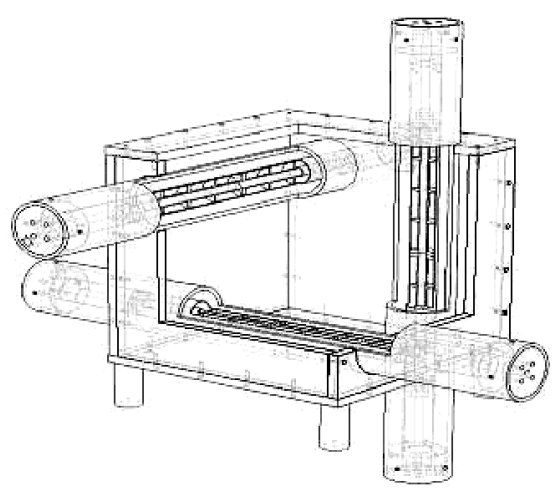

Fig. 2. Three-axes search coil magnetometer for the measurement of the magnetic field in the frequency band [0.001-100] Hz. The instrumental sensitivity is $50 \mathrm{fT} / \sqrt{\mathrm{Hz}}$.

electrical conductivity of the Earth's crust, and the study of the electromagnetic signals generated in the interior of the Earth. The data col- lected in the network stations will allow us to realize environmental electromagnetic tomography through the representation of the electromagnetic fields distribution in the time, in the frequency and in the space domains. When all stations will be in operation the wide-band interferometry technique will be applied using the simultaneous observations of the electromagnetic field measured in the network stations (Palangio et al., 2008). This technique is a good investigation tool to obtain three-dimensional maps of the electromagnetic signals on a regional scale. Tomographic maps are useful to show the spatial distribution of electromagnetic sources, and to characterize the signals by means of the energy, polarization and spectral content. The data collected in each network station will be processed to study the structure of ground electric conductivity, the electromagnetic phenomena connected with the seismic activity, the separation of the electromagnetic fields originated in the interior of the Earth, and the study of the electromagnetic phenomena originated in the magnetosphere, ionosphere and in the Earth-ionosphere cavity. Below the Earth-ionosphere cut-off frequency the interferometric approach will allow us to estimate the magnetic inter-station transfer functions to obtain more information about the electric conductivity of the Earth's crust and to locate the electromagnetic sources by means of interferometric methods. The first MEM station has been in operation in the area of the L'Aquila INGV Geomagnetic Observatory $\left(42^{\circ} 23^{\prime} \mathrm{N}\right.$, $13^{\circ} 19^{\prime} \mathrm{E}, 682 \mathrm{~m}$ a.s.1.) from May the $1^{\text {st }}, 2005$. Within the middle of 2008 two new stations will be activated in Barete $\left(42^{\circ} 30^{\prime} \mathrm{N}, 13^{\circ} 16^{\prime} \mathrm{E}\right.$, $930 \mathrm{~m}$ a.s.1.) and Duronia $\left(41^{\circ} 39^{\prime} \mathrm{N}, 14^{\circ} 28^{\prime} \mathrm{E}\right.$, $910 \mathrm{~m}$ a.s.1.). Figure 1 shows the locations in Central Italy of the first three MEM stations. In the future new stations will be added to the network to cover most part of Central Italy. In each network station the three components of the magnetic field, the three components of the atmospheric electric field and the two components of the telluric field will be measured. The magnetic field will be measured by induction coil magnetometers. The atmospheric electric field will be measured by the electrometer shown in fig. 3 and the telluric field will be 


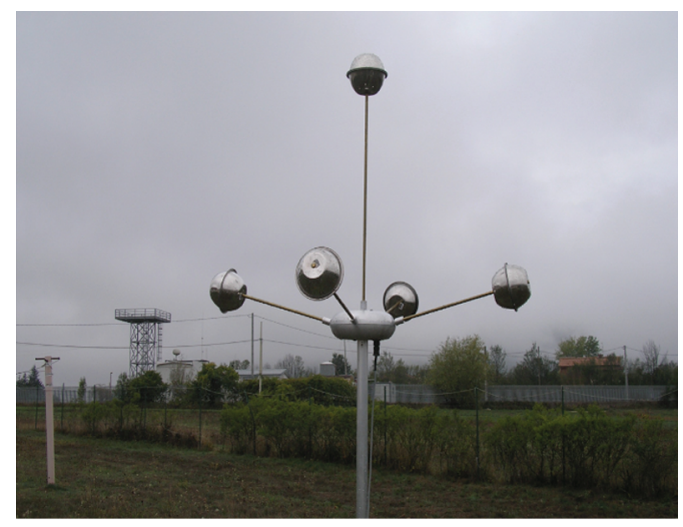

Fig. 3. Three-axes electrometer for the measurement of the atmospheric electric field in the frequency band $[1 \mathrm{~Hz}-100 \mathrm{kHz}]$. The instrumental sensitivity is $1 \mu V /(m \sqrt{H z})$.

measured by two orthogonal non-polarizable electrodes placed at a distance of $140 \mathrm{~m}$. The network configuration is needed to separate the natural electromagnetic fields from the artificial ones and to separate the electromagnetic fields originated in the interior of the Earth from those generated by an external source. The technological objectives of the project are the development of the instrumentation installed in each network station and the know-how transfer to the industry. Figures 2 and 3 show two examples of the instruments developed in the frame of the project. The industrialization of these instruments has been realized by the Italian Geomagnetic System industry. In the Earth-ionosphere cavity there are a myriad of electromagnetic natural and artificial signals produced by a huge number of sources (Palangio, 1993). The spectrum of these signals spreads twelve orders of magnitude in the frequencies domain, and fourteen orders of magnitude in the energy domain (Lanzerotti et al., 1990). Taking into account the multiplicity of the parameters that must be measured, the level of instrumental noise needs to be so low to detect the weakest electromagnetic natural signals. The spectral density of the feeblest natural signal is about [510] $f T / \sqrt{\mathrm{Hz}}$ above the Earth-ionosphere cutoff frequency $(1700 \mathrm{~Hz})$, so the background noise level of the developed instrumentation must be at least [1-2] $f T / \sqrt{\mathrm{Hz}}$. Moreover, taking into account the great variability of the electromagnetic fields involved, a 24 bit A/D converter is used. Using the magnetic and electrical fields measured on the surface of the Earth an impedance tensor can be estimated. The impedance tensor represents the apparent resistivity and phase shift between both the fields as function of the frequency. In conclusion we consider three research areas in the electromagnetic monitoring of geodynamical processes. The first is the determination of the distribution of the electromagnetic noise strength due to the underground sources by means of the data set collected in the interferometric array. The second is the long-term monitoring of the seven rotational invariant parameters of the impedance tensor. The third is the study of the long term monitoring of the magnetic inter-station transfer functions.

\section{Analysis of the signals originated in the Earth interior}

The electromagnetic monitoring of the geodynamic processes is based on two different aspects (Svetov et al., 1997). The first concerns the monitoring of the time variation of the ground electric conductivity due to tectonic stress variations of the rocks in the seismo-genetic area (Meloni et al., 1996; Teisseyre and Ernst, 2002). The second is the monitoring of the electromagnetic fields generated by the different mechanisms of the electro-mechanic energy conversion (Ernst et al., 1997). These mechanisms depend on the rock characteristics (Molchanov and Hayakawa, 1995; Frid et al., 2000). The first point can be studied by the long-term monitoring of the magnetic transfer functions and the elements of the impedance tensor (Spitz, 1985). The knowledge of the underground resistivity structure is an essential requirement to study the electromagnetic manifestations linked to earthquakes. Figure 4 shows the ground electric resistivity profile calculated for L'Aquila station by means of the single station magnetotelluric tensor evaluation. The profile was obtained during May 2005 and the sig- 


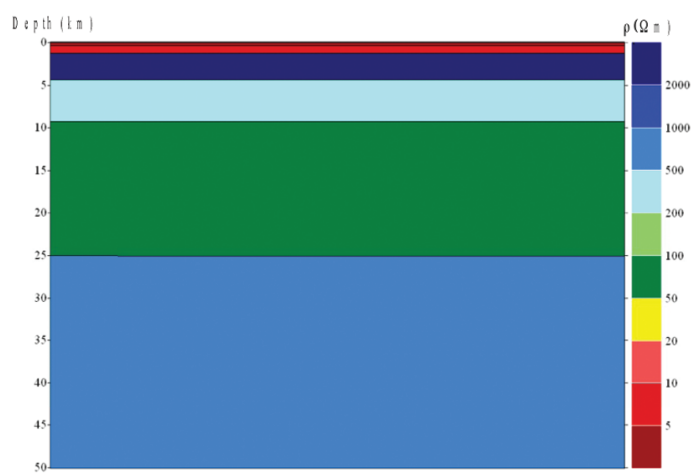

Fig. 4. 1D resistivity model structure of the subsoil in the area of L'Aquila Observatory.

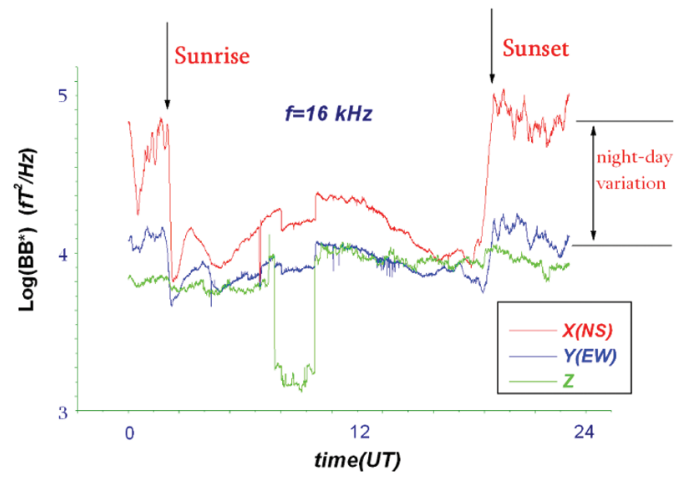

Fig. 5. Large electromagnetic anomaly occurred in L'Aquila on March 14, 2006 between 8:00 and 10:00 UT. The anomaly is probably due to a local electromagnetic source. $B$ represents each of the components $(X, Y, Z)$ of the magnetic field.

nal was collected for fifteen days. To obtain the resistivity profile we used a standard Occam 1D inversion code (Constable et al., 1987), using for the inversion both the apparent resistivities and the phases. Only the two horizontal components of magnetic field and the two horizontal components of the telluric field are used in the calculation. To estimate the transfer functions we used the Egbert algorithm working out both the impedances and the magnetic response functions. In the elaboration, the whole dataset was divided into intervals 4096s long. The final resistivity profile was obtained averaging all the profiles obtained. Concerning the second point we have to consider that the observed signal is the convolution of the source-time-functions and the Earth's impulse response functions. The knowledge of the response functions is a fundamental point for this kind of investigations. Another aspect concerns the structure of the electromagnetic fields, both natural and artificial, in the Earth-ionosphere cavity which changes in a complex way in space and time. The measured signals on the surface of the Earth are given by an overlap of a myriad of waves coming to the measurement point. The separation of the environmental noise and the signals originated in the interior of the Earth was performed by the analysis of the Poynting vector on the residual non-inductive field. Some studies report magnetic anomalies related to the seismic activity. The frequency band of these observations is extremely wide, ranging from $\mathrm{mHz}$ to $\mathrm{MHz}$ (Molchanov et al., 1992). The [0.001-1000] Hz band can be related to linear processes (Molchanov and Hayakawa, 1995), while the [1000 $\mathrm{Hz}-50 \mathrm{kHz}$ ] band can be related to non linear process in the electro-mechanic energy conversion (Parrot et al., 1993). In any case, taking into account the attenuation of the crossed medium on the electromagnetic signals, it is reasonable to observe electromagnetic signals in the band [0.001-1000] Hz. In this frequency band, according the conditions of (Kawate et al, 1998), the amplitude of the electromagnetic signals that reach the surface of the Earth could be sufficient to discriminate it from the background noise (Johnston, 1984). So, this frequency band can be considered the interval in which to focus the research activity. Another interesting band is [1-5] kHz, the so-called dead band (Dea et al., 1993). In this frequency interval the attenuation is high because of the characteristics of the crossed rocks. Moreover in this interval the environmental noise level is low because this is the Earth-ionosphere cut-off band (Meloni et al., 1992). Figure 5 represents an example of a large electromagnetic anomaly measured in L'Aquila on March 14, 2006 between 8:00 and 10:00 UT. The figure shows the three components of the magnetic field in a fre- 
quency band $6 \mathrm{kHz}$ wide around $16 \mathrm{kHz}$. This anomaly cannot be generated or reflected by the ionosphere because the field components do not fulfil the Earth's boundary conditions. Moreover, this anomaly cannot be generated in the interior of the Earth because the skin depth is less than the thickness of the high conductivity sheet in the superficial part of the crust. Probably this anomaly is due to a local electromagnetic source. On the surface of the Earth the generic component $G$, electric or magnetic, of the measured field is due to the superimposition of different terms

$$
G_{m}=G_{e}+G_{i}+G_{s}
$$

where $G_{m}$ is the measured field and $G_{e}, G_{i}$ and $G_{s}$ are respectively the field of external origin, the field induced by the underground currents that $G_{e}$ produces, and the field originated in the interior of the Earth. Only the fields $G_{e}$ and $G_{i}$ can be linked by the Maxwell equations, whereas $G_{s}$ is completely unconnected with the previous fields. In the frequency band [0.01-1] Hz only the one mode prevails, or at least the two modes, of the electromagnetic source. As a rule the link between the inductive field, the field external to the interior of the Earth, and the induced field is outlined by means of the tensor $\boldsymbol{T}$ that represents the properties of the Earth's interior. This tensor can be written as

$$
\begin{aligned}
& \left|E_{x i} E_{y i} E_{z i} H_{x i} H_{y i} H_{z i}\right|^{\prime}= \\
& \left|T_{i j}\right| \cdot\left|E_{x e} E_{y e} E_{z e} H_{x e} H_{y e} H_{z e}\right|^{\prime}
\end{aligned}
$$

In the eq. (2.2) the subscript $i$ and $e$ refer respectively to the induced field and to the inductive field; $\boldsymbol{E}$ is the electric field, $\boldsymbol{H}$ is the magnetic field. As we know only the measured fields $G_{m}$, the eq. (2.1) is apparently unresolved. To resolve the problem, the eq. (2.2) can be simplified considering the next five approximations:

1) quasi stationary approximation: the displacement currents can be neglected respect to the conductivity currents. The propagation of the electromagnetic fields through the Earth can be treated as a diffusive process.

2) the vertical component $H_{z e}$ of the external magnetic field can be neglected respect to the vertical component $H_{z i}$ of the induced field, so the vertical component $H_{z m}$ of the measured magnetic field is

$$
H_{z m}=H_{z i}+H_{z e}+H_{z s} \approx H_{z i}+H_{z s}
$$

3 ) in the case of a subsoil with a low conductivity, the horizontal components $H_{x i}$ and $H_{y i}$ of the induced magnetic field can be neglected respect to the horizontal components $H_{x e}$ and $H_{y e}$ of the external field, so the horizontal components of the measured magnetic field are

$$
\begin{aligned}
& H_{x m}=H_{x e}+H_{x i}+H_{x s} \approx H_{x e}+H_{x s} \\
& H_{y m}=H_{y e}+H_{y i}+H_{y s} \approx H_{y e}+H_{y s}
\end{aligned}
$$

4) the external magnetic field does not have significant horizontal gradients on the incidence surface

$$
\nabla \cdot H_{e} \approx 0
$$

5) the electric vertical field $E_{z i}$ induced in the crust of the Earth can be neglected respect to the horizontal components of the electric field, because of the Earth's boundary conditions $E_{x e} \approx 0$ and $E_{y e} \approx 0$.

With these assumptions, the eq. (2.2) becomes a simplified relation between the measured quantities. So, we are able to calculate the part of the tensor $\boldsymbol{T}$ reported in the following equation:

$$
\left|\begin{array}{l}
\left\langle E_{x m}(\omega)\right\rangle \\
\left\langle E_{y m}(\omega)\right\rangle \\
\left\langle H_{z m}(\omega)\right\rangle
\end{array}\right|=\left|\begin{array}{ll}
T_{14}(\omega) & T_{15}(\omega) \\
T_{24}(\omega) & T_{25}(\omega) \\
T_{64}(\omega) & T_{65}(\omega)
\end{array}\right| \cdot\left|\begin{array}{l}
\left\langle H_{x m}(\omega)\right\rangle \\
\left\langle H_{y m}(\omega)\right\rangle
\end{array}\right|
$$

In the eq. (2.7) the inductive field is represented by the horizontal components of the measured magnetic field. These components of the tensor $\boldsymbol{T}$ allow us to calculate, in the time domain, the six impulse response functions $I_{1}(t)$, $I_{2}(t), I_{3}(t), I_{4}(t), I_{5}(t)$ and $I_{6}(t)$ (Krzysztof, 2004). These functions allow us to calculate the fields originated in the interior of the Earth by means of the convolution of the $I_{i}(t)$ functions and the measured fields (Banks et al., 1993). The components of the inner fields can be obtained by the difference between the measured fields and 


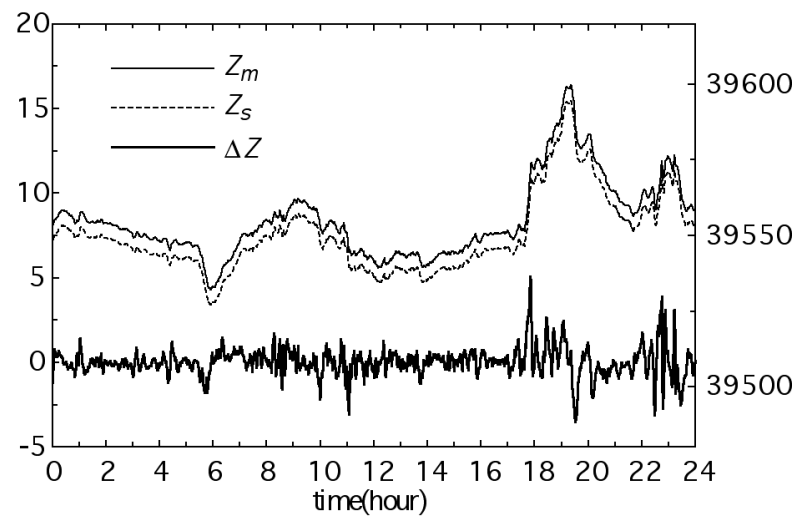

Fig. 6. Evaluation of the reconstructed magnetic field by the convolution of the horizontal field with the Earth's impulse response functions. The vertical scale corresponds only to the difference $\Delta Z$. The measured and the reconstructed vertical components are scaled for a best understanding.

the synthetic fields generated by the convolution of the impulse response functions:

$$
\begin{aligned}
& H_{x s}(t)=H_{x m}-I_{1}(t) \otimes H_{x m}-I_{2}(t) \otimes H_{y m} \\
& H_{y s}(t)=H_{y m}-I_{3}(t) \otimes H_{x m}-I_{4}(t) \otimes H_{y m} \\
& H_{z s}(t)=H_{z m}-I_{5}(t) \otimes H_{x m}-I_{6}(t) \otimes H_{y m} \\
& E_{x s}(t)=E_{x m}-I_{7}(t) \otimes H_{x m}-I_{8}(t) \otimes H_{y m} \\
& E_{y s}(t)=E_{y m}-I_{9}(t) \otimes H_{x m}-I_{10}(t) \otimes H_{y m}
\end{aligned}
$$

Figure 6 shows the vertical component of the measured signal, the signal reconstructed from a single station data set using inductive methods, and the difference between them. The difference between the two signals could be due to underground sources. The other four impulse response functions $I_{7}(t), I_{8}(t), I_{9}(t)$, and $I_{10}(t)$ can be evaluated by the inter-station transfer functions (Harada et al., 2004). In this case the approximation 3 is not considered. However, the difference could also be generated on the surface of the Earth near the measurement point, but these proximal signals do not produce significant inductive effects. Their contribution on the impulse response functions is null (Palangio et al., 1991). To solve this ambiguity we can calculate the Poynting vector from the residual field to obtain the arrival direction of the residual signals and to draw out the electromagnetic fields originated in the interior of the Earth. By the time dependent expression of the Poynting vector $\boldsymbol{P}(t)=\boldsymbol{E}(t) \times \boldsymbol{H}(t)$, we obtain the three components $P_{x} P_{y} P_{z}$ on the surface of the Earth

$$
\begin{aligned}
& P_{x s}(t)=\left(\begin{array}{ll}
E_{y s} & E_{z s} \\
H_{y s} & H_{z s}
\end{array}\right) \\
& P_{y s}(t)=-\left(\begin{array}{ll}
E_{x s} & E_{z s} \\
H_{x s} & H_{z s}
\end{array}\right) \\
& P_{z s}(t)=\left(\begin{array}{ll}
E_{x s} & E_{y s} \\
H_{x s} & H_{y s}
\end{array}\right)
\end{aligned}
$$

The $x$ direction corresponds with the geographic north, the $y$ direction corresponds with the geographic east, and the $z$ is directed toward the centre of the Earth. Since $E_{z s} \sim 0$, eq. (2.9) becomes

$$
\begin{aligned}
& P_{x s}=E_{y s} H_{z s} \\
& P_{y s}=-E_{x s} H_{z s} \\
& P_{z s}=\left(E_{x s} H_{y s}-E_{y s} H_{x s}\right)
\end{aligned}
$$

In the frequency domain, these functions are complex quantities. Generally we have two vectors, one real and the other imaginary, orien- 
tated in different directions. Some contributions to the Poynting vector could be due to the reflected signals. So, we must consider the electromagnetic field component which is reflected by the surface of the Earth. If $P_{r e f}$ and $P_{i n c}$ are respectively the flux of the reflected wave and the flux of the incident wave, negative values of the transmission factor $T=1-P_{\text {ref }} / P_{\text {inc }}$ could in-

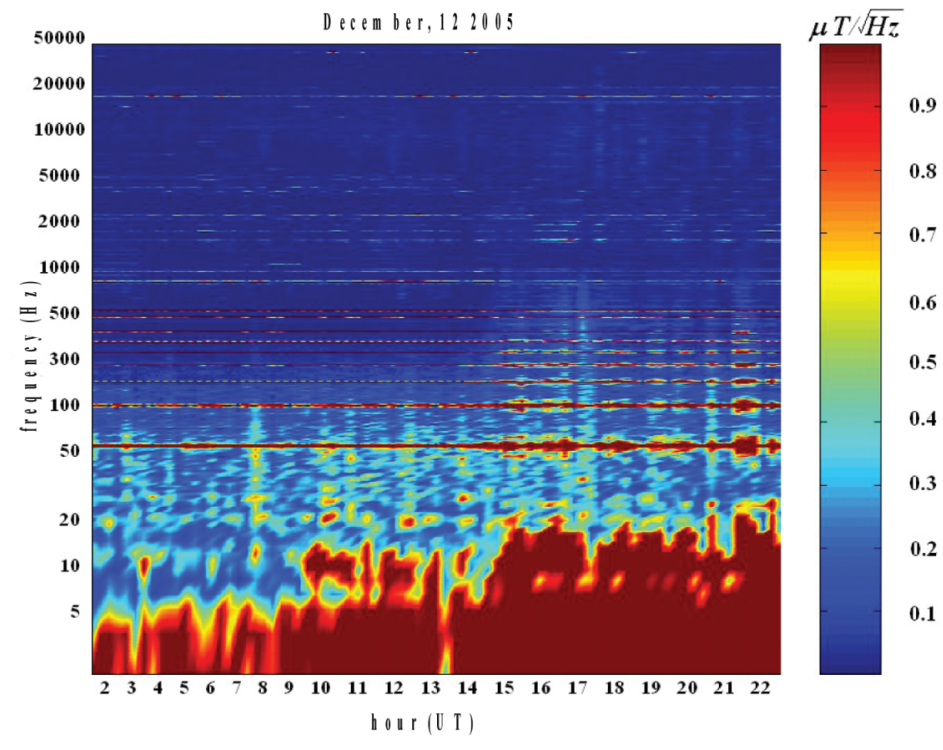

Fig. 7. Spectrogram of the horizontal magnetic field in the frequency band from $[1 \mathrm{~Hz}-50 \mathrm{kHz}]$.

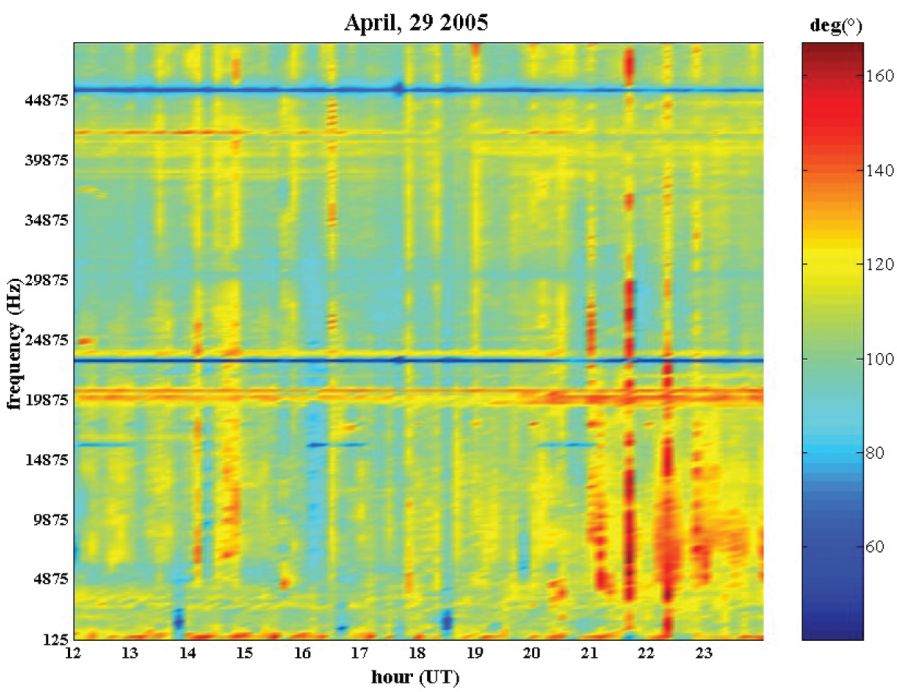

Fig. 8. Zenithal component of the Poynting vector. 
dicate an electromagnetic source inside the Earth. Considering a homogeneous plane wave incident on the surface of the Earth, the impedance of the Earth's surface can be written as $Z_{s} \approx \sqrt{j \omega \mu /(\sigma+j \omega \varepsilon)}$. If $Z_{s}$ is isotropic and uniform, then the electric and the magnetic fields are related each other by $E=H Z_{s}$. Generally, the impedance of the Earth's surface is a complex function which could be considered consisting by a resistive component $R$ and a reactive component $X_{L}$ of equal magnitude, and can be written as $Z_{s}=R+j X_{\mathrm{L}}$. If $\sigma>>\omega \varepsilon$ then $Z_{s} \approx \sqrt{\omega \rho \mu / 2}+j \sqrt{\omega \rho \mu / 2} ;$ this means that the fields $\boldsymbol{E}$ and $\boldsymbol{H}$ are $45^{\circ}$ out of phase. In the frequency band $[0.001 \mathrm{~Hz}-5 \mathrm{kHz}]$, considering the integrated values of $\rho(\omega)$ at each frequencies, the impedance $Z_{s}$ lies in the interval [0.001-4] $\Omega \mathrm{m}$. Concerning the signals originated in the ionosphere, the wave impedance $Z_{w}$ of the signal incident on the surface of the Earth depends on the frequency, on the impedance of the ionosphere and on the distance between the Earth and the ionosphere. $Z_{w}$ represents the impedance of the waves generated in the dynamo region of the ionosphere, prior to reach the earth surface. Considering a very simple model of isotropic ionosphere, like a metallic conductor, with a conductivity independent on the frequency below the lowest plasma frequency in the dynamo region, in the range of frequency $[1 \mathrm{mHz}-0.5 \mathrm{~Hz}]$ (strong near field condition), the wave impedance is approximately of the same order of the impedance of the Earth's surface. In absence of non inductive signals, the average flux transferred through the surface of the Earth is equal to $P_{\text {inc }}-P_{\text {ref }}$, so the electric and magnetic signals measured are

$$
\begin{gathered}
E_{m}=E_{i n c}+E_{r e f}=Z_{w} H_{i n c}-Z_{s} H_{r e f} \\
H_{m}=H_{i n c}+H_{r e f}
\end{gathered}
$$

If we consider a simplified case, a polarized field $E_{y}=0, E_{z}=0, H_{x}=0$ and $H_{z}=0$, consequently $P_{x}=0, P_{y}=0$ and $P_{z}=E_{x} H_{y}$. Therefore

$$
\frac{P_{\text {ref }}}{P_{\text {inc }}}=\frac{Z_{s}\left(Z_{w} H_{y}-E_{x}\right)^{2}}{Z_{w}\left(E_{x}+Z_{s} H_{w}\right)^{2}}
$$

where $H_{y}$ and $E_{x}$ are the measured fields. If, $Z_{s} \approx$ $\approx Z_{w}, E_{x} \approx Z_{s} H_{y}$ therefore $P_{\text {ref }} / P_{\text {inc }} \approx 0$ and $T \approx 1$. In this case almost the whole energy penetrates in the Earth. On the contrary, in the frequency band $[0.5 \mathrm{~Hz}-5 \mathrm{kHz}] Z_{w}>Z_{s}$, so that a reflected signal component is present. Generally, the signals transmitted inside the Earth are

$$
\begin{aligned}
H_{t} & \approx 2\left[Z_{w} /\left(Z_{w}+Z_{s}\right)\right] H_{i n c} \\
E_{t} & \approx 2\left[Z_{s} /\left(Z_{w}+Z_{s}\right)\right] E_{i n c}
\end{aligned}
$$

Because $Z_{w}>Z_{s}$, a fraction of the incident signal is reflected by the surface of the Earth, so the Poynting vector has a component directed away from the surface of the Earth. This could be considered as a signal originated in the lithosphere. In order to avoid this effect in the upper part of the spectrum, it is necessary to evaluate the impedance of the waves and the media using models of the conductivities of the Earth and the ionosphere. Figures 7, and 8 show two examples concerning the applications of the electromagnetic data collected in the MEM station of L'Aquila. We stress that the data sets reported were chosen in the days with low atmospheric noise. Figure 7 shows a 2D representation of the horizontal magnetic field in the frequency and the time domains. The plot shows stationary artificial signals $(50 \mathrm{~Hz}$ and its harmonics till few $\mathrm{kHz}$ ) and a lot of signals due to non stationary sources. Figure 8 shows the representation of the zenithal component of the Poynting vector as function of the time and the frequency. From the figure can be noted that the arrival direction is ranging mainly from $60^{\circ}$ to $140^{\circ}$ showing that the major part of the signals reaches the measurement point by ionospheric reflection. Regarding the signals originated in the interior of the Earth we report an example of the long-term analysis of the characteristics of the magnetic induction vectors in the lower frequency band, where the reflection problems are not present. In order to discriminate the anomalous variations of the underground conductivity, this analysis tends to investigate the normal behaviour of induction vectors parameters when no large crustal changes due to geodynamical processes are present. From the eq. (2.7) we can estimate $T_{64}(\omega)$ and $T_{65}(\omega)$ which generally are complex functions. They are time invariant functions if 
the electric resistivity of the ground is stable, namely when there are no large crustal changes due to geodynamical processes. Starting from $T_{64}(\omega)$ and $T_{65}(\omega)$, we can calculate both the real and the imaginary induction vectors modules.

$$
\begin{aligned}
& |T|_{\text {real }}=\sqrt{\left[\operatorname{Re}\left(T_{64}(\omega)\right)\right]^{2}+\left[\operatorname{Re}\left(T_{65}(\omega)\right)\right]^{2}} \\
& |T|_{\text {imag }}=\sqrt{\left[\operatorname{Im}\left(T_{64}(\omega)\right)\right]^{2}+\left[\operatorname{Im}\left(T_{65}(\omega)\right)\right]^{2}}
\end{aligned}
$$

and the real and imaginary phases

$$
\begin{aligned}
& \Theta_{\text {real }}=a \tan \left[\operatorname{Re}\left(T_{65}(\omega)\right) / \operatorname{Re}\left(T_{64}(\omega)\right)\right] \\
& \Theta_{\text {imag }}=a \tan \left[\operatorname{Im}\left(T_{65}(\omega)\right) / \operatorname{Im}\left(T_{64}(\omega)\right)\right]
\end{aligned}
$$

Figures 9,10 and 11 show the time variations of the magnetic induction vectors during 2006 for L'Aquila MEM site in three frequency bands: [0.001-0.010] Hz, [0.05-0.10] Hz and [0.15$0.20 \mathrm{Jzz}$. The considered frequency intervals can be related to the whole crust in term of skin depth. In Central Italy the major tectonic activity is concentrated in the underground depth range of about $5-15 \mathrm{~km}$. In this area the direction of the active faults is roughly NW-SE (see fig. 1). In figures 10 and 11 it can be noted that the direction of the real vector points toward the tectonic alignment, perpendicularly to the breaking planes of the faults structure. The imaginary vector has the same direction of the real vector. This suggests almost a $2 \mathrm{D}$ resistivity structure in the middle part of the crust. Figure 9 shows the time variations of the magnetic induction vectors in the frequency band [0.001-0.010] Hz. This fre-
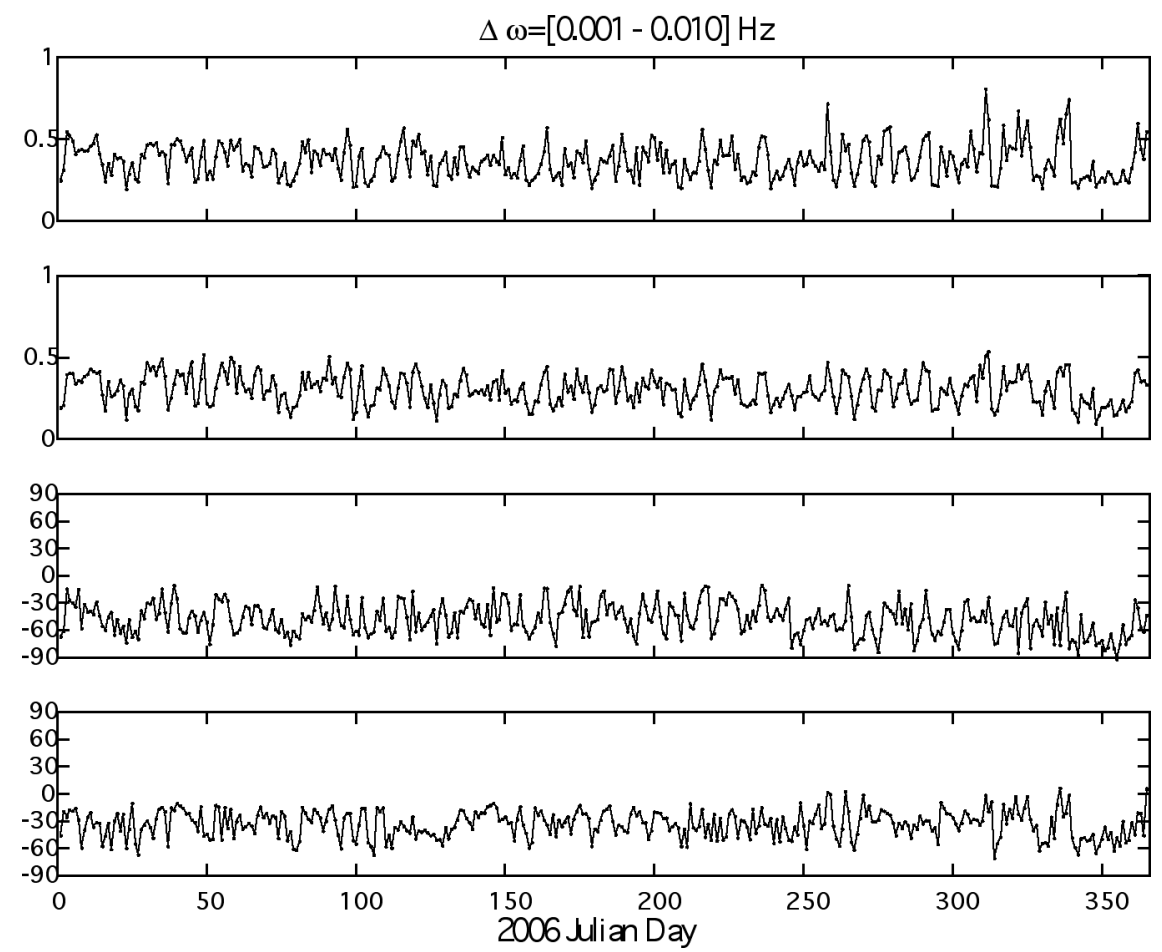

Fig. 9. Daily mean of the magnetic induction vectors calculated for L'Aquila data set in the frequency band [0.001-0.010] Hz. The time period cover the whole 2006. 

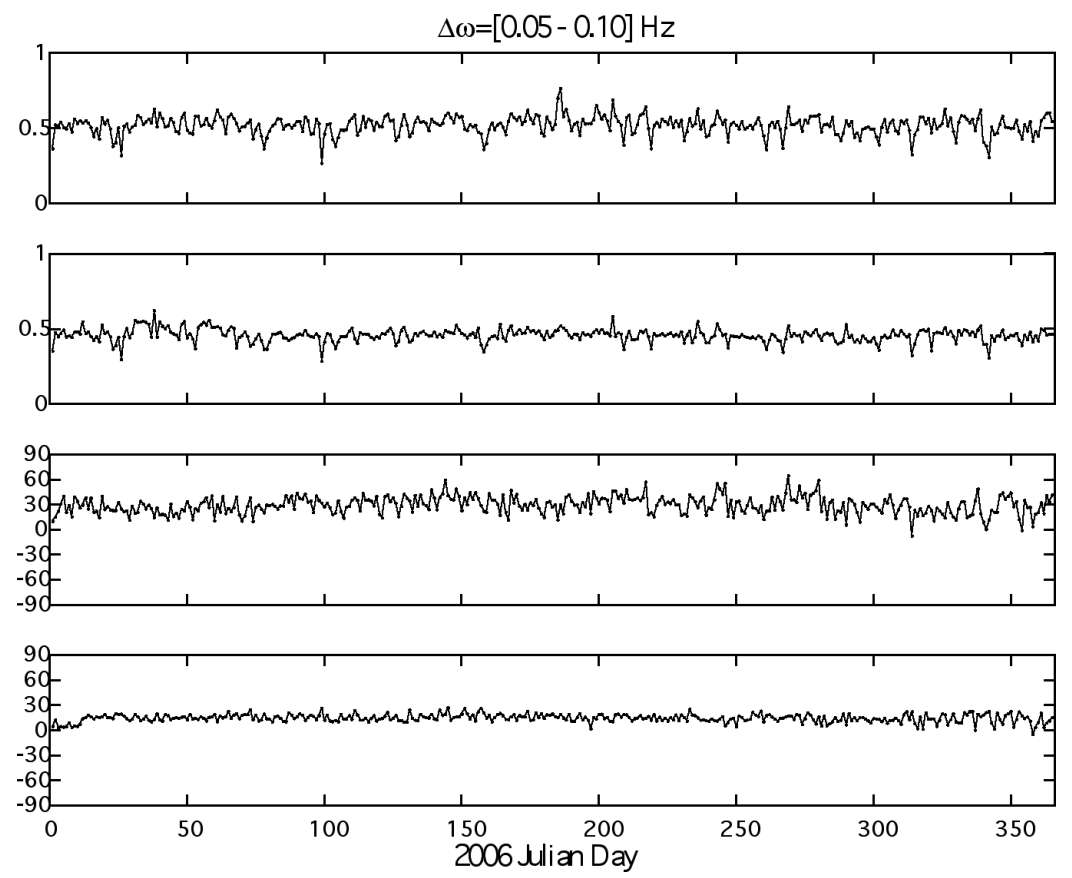

Fig. 10. As fig. 12 for the frequency band $[0.05-0.10] \mathrm{Hz}$.
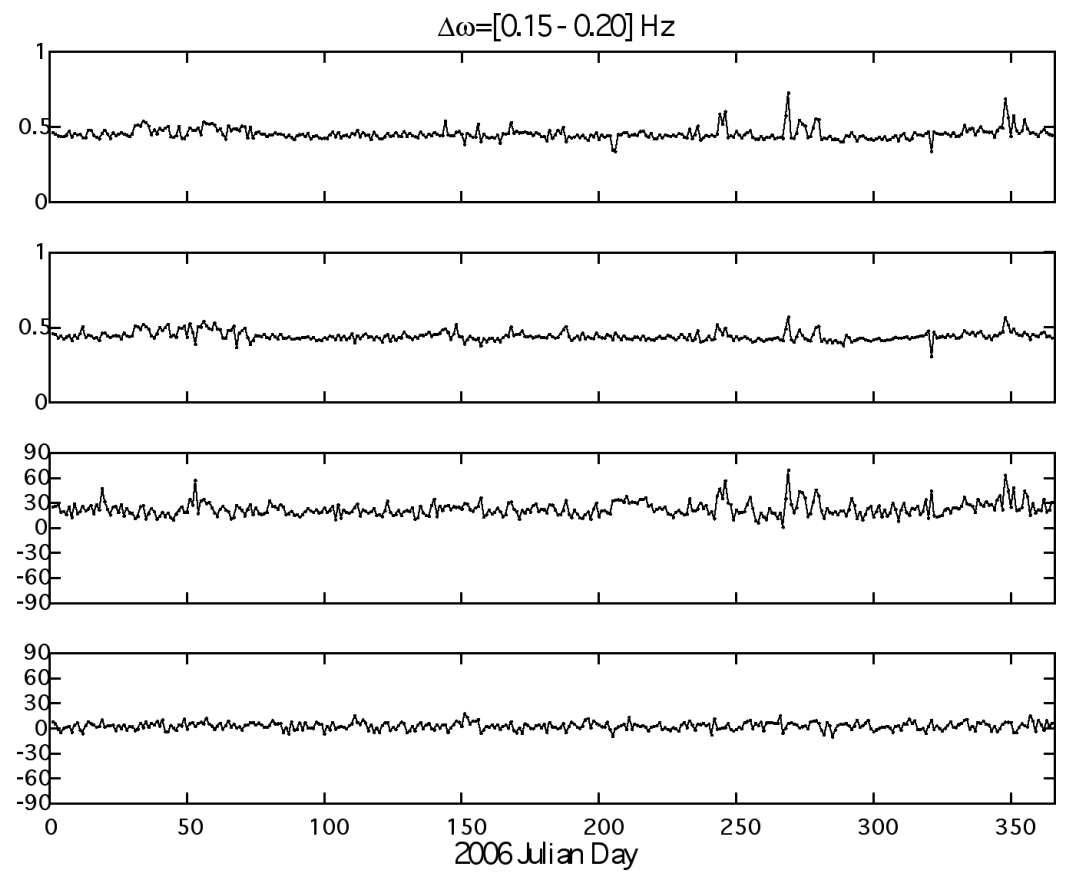

Fig. 11. As fig. 12 for the frequency band [0.15-0.20] Hz. 
quency interval can be related to the deeper part of the crust. The figure shows that the directions of the real and imaginary vectors are quite different respect to the directions of the vectors in the band previously reported. This feature reflects a different orientation of the lateral discontinuity of the resistivity structure. In any case, the real and the imaginary vectors are parallel each other; this clearly shows again a 2D resistivity structure in the lower part of the crust, but different respect to the resistivity structure of the middle part of the crust. The induction vectors shown in figs. 9, 10 and 11 do not show anomalous behaviour during the reported period. In any case no significant seismic activity was recorded in Central Italy in this period, so no significant anomalies in the local geomagnetic field are expected.

\section{Conclusions}

We have described the scientific and the technological objectives of the MEM project and the methodologies proposed to study the electromagnetic signals in the Earth-ionosphere cavity. Some methodologies have been proposed to study the electromagnetic sources by means of the wide band interferometry technique. We have also reported some results obtained from the data set of the first station of the network installed in the area of the L'Aquila INGV Observatory. Some examples, such as the Poynting vector calculation in the whole frequency band, the energy distribution in time and frequency domain and the annual averaged power as function of frequency are reported. Using the single station magnetotelluric approach we obtained some valuable information on the underground resistivity structure in the area of the measurement station. We also show an example of the reconstruction of the vertical component of the magnetic field by means of a single site impulse response functions. Concerning the study of the magnetic signals linked to the tectonic activity we have reported an example of the long term behaviour of the magnetic induction vector characteristics in the lower frequency band $[0.001-0.5] \mathrm{Hz}$, showing their normal behaviour when no large crustal changes due to geodynamical processes are present. We will improve this kind of analysis for a longer period of time in order to understand the nature of the relationship between the intensity of the anomalous bahaviour of the transfer functions and the parameters of the earthquakes such as the magnitude, the hypocentral depth and the distance from the measure site.

\section{Acknowledgements}

The author thanks the administrative staff of the INGV L'Aquila Observatory for the basic support in the activity. This work was supported by the MEM Project (Interreg IIIA Adriatic Cross Border Programme).

\section{REFERENCES}

Banks, R.J., A.A.K. Irving and D.W. Liverlybrooks (1993): The simulation of magnetic variation anomalies using single-station data, Phys. Earth Planet. In., 81, 85-98.

Constable, S.C., R.L. Parker and C.G. Constable (1987): Occam's inversion; a practical algorithm for the inversion of electromagnetic data, Geophysics, $\mathbf{5 2}$, 289-300.

DeA, J.Y., P.M. HANSEN and W.M. Boerner (1993): Longterm ELF background noise measurements, the existence of window regions, and applications to earthquake precursor emission studies, Phys. Earth Planet. In., 77, 109-125.

Ernst, T., R. Teisseyre, A. Meloni, P. Palangio and M. MARCHETTI (1997): Magnetic variation analysis for June 1993 seismic events in Central Italy, Ann. Geofis., 40 (2), 539-545.

Frid, V., D. BAHAT, J. Goldbaum and A. RabinOvich (2000): Experimental and theoretical investigations of electromagnetic radiation induced by rock fracture, $I S$ rael J. Earth Sci., 49, 9-19.

HARAdA M., K. HATTORI and N. IsEZAKI (2004): Transfer function approach to signal discrimination of ULF geomagnetic data, Phys. Chem. Earth, 29, 409-417.

Kawate, R., O.A. Molchanov and M. Hayakawa (1998): Ultra-lowfrequency magnetic fields during the Guam earthquake of 8 August 1993 and their interpretation, Phys. Earth Planet. In., 105, 229-238.

KRZYSZTOF, N. (2004): Estimation of magnetotelluric transfer functions in the time domain over wide frequency band, Geophys. J. Int., 158, 32-42.

Johnston, M.J.S. (1984): Precision of magnetic measurements in a tectonically active region, J. Geomagn. Geoelectr., 36, 83-95.

LANZEROtTI, L.J., C.G. MaClenNAN and A.C. Fraser-SMith (1990): Background magnetic spectra: $\sim 10 \mathrm{E}-5$ to $\sim 10 \mathrm{E}+5 \mathrm{~Hz}$, Geophys. Res. Lett., 17 (10), 1593-1596. 
Meloni A., P. Palangio and A.C Fraser Smith (1992): Some characteristics of the ELF/VLF radio noise measured near L'Aquila, Italy, IEEE T. Antenn. Propag., 40 (2), 12-18.

Meloni, A., P. Palangio, M. Marchetti, R. Teisseyre, T. ERnST and J. MARIANiUK (1996): Central Italy magnetotelluric investigation. Structure and relations to seismic events: analysis of initial data, Ann. Geofis., 39 (1), 159-177.

Molchanov, O.A. and M. Hayakawa, (1995): Generation of ULF electromagnetic emissions by microfracturing, Geophys. Res. Lett., 22, p. 3091-3094.

Molchanov, O.A., Yu.A. Kopytenko, P.M. Voronov, E.A. Kopytenko, T.G. Matiashvili, A.C FraserSMITH and A. BERNARDI (1992): Results of ULF magnetic field measurements near the epicenters of the Spitak $(M s=6.9)$ and Loma Prieta $(M s=7.1)$ earthquakes comparative analysis, Geophys. Res. Lett., 19, 14951498.

Palangio, P. (1993): Radioricezione ELF-VLF, Ann. Geofis., XXXVI (5-6), 99-114.

Palangio, P., M. Marchetti and L. Di Diego (1991): Ru- more elettromagnetico prodotto dalle ferrovie elettrificate. Effetti sulle misure magnetotelluriche e geomagnetiche, Atti del X Convegno Annuale del GNGTS, 126139.

Palangio, P., F. Masci, M. Di Persio and C. Di Lorenzo (2008): Electromagnetic field measuremets in ULFELF-VLF [0.001 Hz-100 kHz] bands, Adv. Geosci., 14, 69-73.

Parrot, M., J. Achache, J. J. Berthelier, E. Blanc, A. Deschamps, F. Lefeuvre, M. MenVielle, J.L. Plantet, P. TARits and J.P. Villian (1993): High-frequency seismo-electromagnetic effects, Phys. Earth Planet. In., 77, 65-83.

SPITZ, S. (1985): The magnetotelluric impedance tensor properties with respect to rotations, Geophysics, 50 (10), 1610-1617.

Svetov, B. S., S. D. KarinskiJ, I. Y. Kuksa and V. I. ODINTSOV (1997): Magnetotelluric monitoring of geodynamic processes, Ann. Geofis., XL (2), 219-237.

TEISSEYRE, R. and T. ERNST (2002): Electromagnetic radiation related to dislocation dynamics in a seismic preparation zone, Ann. Geophys., 45, 393-399. 